\title{
BER Analysis for MPAM Signal Constellations in the Presence of Fading and ADC Quantization Noise
}

\author{
Umar H. Rizvi, Student Member, IEEE, Gerard J. M. Janssen, Member, IEEE, \\ and Jos H. Weber, Senior Member, IEEE
}

\begin{abstract}
In this letter, closed-form expressions for the bit error rate of $M$-ary pulse amplitude modulated signal constellations as a function of the analog-to-digital converter word length, the signal-to-noise ratio and the fading distribution, are derived. These results allow for a rapid and accurate evaluation of the system performance when the analog-to-digital converter resolution is limited, as is generally the case in high sampling rate communication systems, and thus provide a useful tool for system design, analysis and optimization.
\end{abstract}

Index Terms-ADC quantization noise, BER analysis, Nakagami fading and RF system design.

\section{INTRODUCTION}

D IRECT conversion transceivers employing single-carrier (SC) schemes with directional antennas to counter intersymbol-interference (ISI) are a promising alternative to multicarrier (MC) schemes, for high data rate communication systems operating in the $60 \mathrm{GHz}$ and $100 \mathrm{GHz}$ regime. Inphase and quadrature-phase (I/Q) imbalance is one of the major problems associated with direct conversion transceivers [1] which can be alleviated to an extent by employing one dimensional signal constellations [2]. It is well known that the power consumption in an analog-to-digital converter (ADC) is directly proportional to $F_{s} \cdot 2^{N}$, where $F_{s}$ denotes the sampling frequency and $N$ represents the ADC word length. Thus with sampling rates in the range of Giga samples per second, $N$ must be kept as small as possible. Monte Carlo simulations are often used to evaluate the system performance in terms of average probability of error in the presence of limited ADC resolution. In [3] an analytical expression for the BER of orthogonal frequency division multiplexing (OFDM) based MC schemes is presented, which is based on the assumption that ADC quantization noise $(\mathrm{QN})$ is Gaussian distributed. This assumption is valid only when the OFDM system employs a large number of sub-carriers and in general is not true for SC schemes.

In this letter, we derive closed form expressions for the bit error rate (BER) of Gray coded $M$-ary pulse amplitude modulated (MPAM) signal constellations for SC transmission in the presence of ADC QN over additive white Gaussian noise (AWGN) and fading channels. With the novel derived expressions, 2PAM (i.e, binary phase shift keying (BPSK)) and 4PAM are used as examples for the performance evaluation

Manuscript received July 30, 2009. The associate editor coordinating the review of this letter and approving it for publication was G. K. Karagiannidis.

The authors are with IRCTR/CWPC, Wireless and Mobile Communications Group, Faculty of Electrical Engineering, Mathematics and Computer Science, EEMCS, Delft University of Technology, 2628 CD Delft, The Netherlands (e-mail: \{u.h.rizvi, g.janssen, j.h.weber\}@ewi.tudelft.nl).

Digital Object Identifier 10.1109/LCOMM.2009.091586 in various fading cases. For fading analysis the Nakagami distribution is assumed which covers line-of-sight as well as non-line-of-sight channels. To the best of our knowledge, no closed form expressions for MPAM schemes in the presence of ADC QN, AWGN and fading have been reported in the literature.

The remainder of the letter is organized as follows. In Section II, the system model is introduced. The closed form expressions for the BER are presented in Section III and numerical results are given in Section IV. Conclusions are drawn in Section V.

\section{SYSTEM MODEL}

The real baseband equivalent received signal of an MPAM communication system, after coherent detection, operating under the influence of fading $h$, AWGN $n$ and in the presence of ADC QN $u$ is given as $y=h s+n+u=h s+z$, where $s$ denotes the transmitted symbol chosen from the signal set $\mathcal{S}=\left\{s_{0}, \cdots, s_{M-1}\right\}$. The AWGN is assumed to be zero mean with variance $\sigma_{n}^{2}$ and power spectral density $N_{0}=2 \sigma_{n}^{2}$. The additive QN $u$ is uniformly distributed between $\left[-V / 2^{N}, V / 2^{N}\right][4$, p.186] and its variance is related with $N$ as $\sigma_{u}^{2}=V^{2} /\left(3 \cdot 2^{2 N}\right)$, where the ADC is assumed to be operating over a voltage range $[-V, V]$ volts. It is assumed that prior to ADC an automatic gain control circuit brings the signal within $[-V, V]$ and there is no clipping. In this letter all signal constellations are assumed to be Gray coded and equally distributed between $[-V, V]$ with an an inter-symbol distance of $d=2 V /(M-1)$, i.e., the $l$-th symbol in the signal constellation set is given as $s_{l}=V(2 l-M+1) /(M-1)$. The average constellation energy is given as $E_{s}=(1 / M) \sum_{l=0}^{M-1} s_{l}^{2}=S_{M} V^{2} /\left(M(M-1)^{2}\right)$, where $S_{M}=\sum_{l=0}^{M-1}(2 l-M+1)^{2}$. Thus we get $V=\sqrt{M E_{s}}(M-1) / \sqrt{S_{M}}$. A system with frequency flat fading is assumed. The independent and identically distributed fading amplitudes $h$ are drawn from a distribution with probability density function (PDF) $p(h)$. Assuming perfect channel state information at the receiver, symbol wise detection is performed, with the most likely transmitted symbol being chosen as the symbol which minimizes the metric $\mathcal{C}\left(y, s_{k}\right)=\left|y-h s_{k}\right|^{2}$ over all $s_{k} \in \mathcal{S}$.

\section{BER ANALYSIS}

The signal-to-noise ratio (SNR) per symbol is defined as $\gamma=E_{s} / N_{0}$. The average energy per bit $E_{b}$ is related with $E_{s}$ as $E_{s}=\log _{2}(M) E_{b}$. The SNR per bit is thus given as $\gamma_{b}=$ $\gamma / \log _{2}(M)=E_{b} / N_{0}$. In the fading case, the instantaneous 


$$
\begin{aligned}
P_{b}= & K_{M} \sum_{k=1}^{\log _{2}(M)} \sum_{i=0}^{\mathcal{L}(k, M)} \mathcal{X}(k, i, M) \cdot Q\left(\sqrt{M \gamma}\left(\frac{2 \cdot \mathcal{Y}(i)+(M-1) \cdot 2^{-N+1}}{\sqrt{2 S_{M}}}\right)\right) \cdot\left(1+\frac{\mathcal{Y}(i) \cdot 2^{N}}{M-1}\right)+ \\
& K_{M} \sum_{k=1}^{\log _{2}(M)} \sum_{i=0}^{\mathcal{L}(k, M)} \mathcal{X}(k, i, M) \cdot Q\left(\sqrt{M \gamma}\left(\frac{2 \cdot \mathcal{Y}(i)-(M-1) \cdot 2^{-N+1}}{\sqrt{2 S_{M}}}\right)\right) \cdot\left(1-\frac{\mathcal{Y}(i) \cdot 2^{N}}{M-1}\right)- \\
& K_{M} \sum_{k=1}^{\log _{2}(M)} \sum_{i=0}^{\mathcal{L}(k, M)} \frac{\mathcal{X}(k, i, M) \cdot \sqrt{S_{M}}}{(M-1) \sqrt{M \pi \gamma} 2^{-N+1}} \cdot\left[e^{-\frac{M \gamma}{S_{M}}\left(\mathcal{Y}(i)+(M-1) \cdot 2^{-N}\right)^{2}}-e^{-\frac{M \gamma}{S_{M}}\left(\mathcal{Y}(i)-(M-1) \cdot 2^{-N}\right)^{2}}\right] .
\end{aligned}
$$

and average SNRs are given as $h^{2} \gamma$ and $E\left[h^{2}\right] \gamma$, respectively, where $E[$.$] denotes the expectation operation. In this letter$ $E\left[h^{2}\right]=1$ is assumed.

\section{A. $A W G N$ and $A D C Q N$}

In [5], the BER for Gray coded MPAM signal constellations in the presence of AWGN is given, which can be generalized for any additive noise $z$ as

$$
P_{b}=2 K_{M} \sum_{k=1}^{\log _{2}(M)} \sum_{i=0}^{\mathcal{L}(k, M)} \mathcal{X}(k, i, M) \int_{\frac{\mathcal{y}(i) d}{2}}^{\infty} q(z) d z,
$$

where $q(z)$ represents the noise PDF and $K_{M}=$ $1 /\left(M \log _{2}(M)\right)$. The modulation and bit dependent functions $\mathcal{L}(k, M), \mathcal{X}(k, i, M)$ and $\mathcal{Y}(i)$ are seen to follow a regular pattern for Gray coded MPAM signal constellations and are given as $\mathcal{L}(k, M)=\left(1-2^{-k}\right) M-1, \mathcal{X}(k, i, M)=$ $(-1)^{\left\lfloor\frac{i \cdot 2^{k-1}}{M}\right\rfloor}\left(2^{k-1}-\left\lfloor\frac{i \cdot 2^{k-1}}{M}+\frac{1}{2}\right\rfloor\right)$ and $\mathcal{Y}(i)=2 i+1[5]$

The combined PDF of a sum of normally $\mathcal{N}\left(0, \sigma_{n}^{2}\right)$ and uniformly $\mathcal{U}\left(0, \sigma_{u}^{2}\right)$ distributed random variables $z=n+u$ $[6, \mathrm{p} .22]$ can be stated as

$q(z)=\frac{2^{N-2}}{V}\left[\operatorname{erf}\left(\frac{z+V \cdot 2^{-N}}{\sqrt{N_{0}}}\right)-\operatorname{erf}\left(\frac{z-V \cdot 2^{-N}}{\sqrt{N_{0}}}\right)\right]$,

where $\operatorname{erf}(x)=\frac{2}{\sqrt{\pi}} \int_{0}^{x} e^{-t^{2}} d t$, which is related to the $Q$ function as $\operatorname{erf}(x)=1-2 Q(\sqrt{2} x)$. Substituting (2) in (1) and solving the integral yields the expression of the BER for AWGN channels in the presence of ADC QN as given in (3). As an illustrative example the BER of 2PAM (BPSK), by substituting $M=2$ in (3) can be written in closed form as

$$
\begin{aligned}
P_{b}= & \left(\frac{1}{2}+2^{N-1}\right) Q\left(\sqrt{2 \gamma}\left(1+2^{-N}\right)\right)+ \\
& \left(\frac{1}{2}-2^{N-1}\right) Q\left(\sqrt{2 \gamma}\left(1-2^{-N}\right)\right)- \\
& \frac{2^{N-2}}{\sqrt{\pi \gamma}}\left[e^{-\gamma\left(1+2^{-N}\right)^{2}}-e^{-\gamma\left(1-2^{-N}\right)^{2}}\right] .
\end{aligned}
$$

For the case when there is no additive quantization noise, i.e., $N \rightarrow \infty$, (4) reduces to $\lim _{N \rightarrow \infty} P_{b}=Q(\sqrt{2 \gamma})$, which is the exact expression for the error probability of BPSK in AWGN channels.

\section{B. Fading, $A W G N$ and $A D C Q N$}

For the case of fading channels and coherent detection the signal constellation is scaled by a factor of $h$. This implies that for a given fading realization $h$, the Euclidean inter-symbol distance is given as $h d$. Therefore, the BER evaluation requires the computation of an average over the fading distribution $p(h)$ as

$$
P_{b}^{F}=\int_{0}^{\infty} P_{b}(h) p(h) d h,
$$

where $P_{b}(h)$ denotes the instantaneous BER for a given fading realization $h$ and is given as

$$
P_{b}(h)=2 K_{M} \sum_{k=1}^{\log _{2}(M)} \sum_{i=0}^{\mathcal{L}(k, M)} \mathcal{X}(k, i, M) \int_{\frac{\mathcal{y}(i) h d}{2}}^{\infty} q(z) d z .
$$

Following the derivation in the last section it can be easily shown that $P_{b}(h)$ can be written as (7) in case $z=n+u$ as before. By substituting $h=(1+x) /(1-x)$ in (5), multiplying with $\sqrt{1-x^{2}} / \sqrt{1-x^{2}}$ and using the GaussChebyshev quadrature (GCQ) [7, p.889, 25.4.38], the BER over fading channels in the presence of AWGN and ADC QN can be approximated as

$$
P_{b}^{F} \approx G_{L} \sum_{l=1}^{L} P_{b}\left(\frac{1+x_{l}}{1-x_{l}}\right) p\left(\frac{1+x_{l}}{1-x_{l}}\right) \frac{\sqrt{1-x_{l}^{2}}}{\left(1-x_{l}\right)^{2}},
$$

where $G_{L}=2 \pi / L, x_{l}=\cos ((2 l-1) \pi / 2 L)$ and $L$ denotes the degree of the Gauss-Chebyshev polynomial. The larger the value of $L$ the closer is the approximation to the actual value. For our analysis a value of $L=16$ was found to be sufficient. The expression given in (8) is in general true for any fading distribution but we restrict our discussion to the Nakagami- $m$ distribution. The PDF of the Nakagami- $m$ distribution is given as

$$
p(h)=\frac{2 m^{m} h^{2 m-1}}{\Gamma(m)} e^{-m h^{2}},
$$

where $\Gamma($.$) denotes the gamma function. It is good to note$ here that the Rayleigh distribution is a special case of the Nakagami- $m(m=1)$ distribution.

\section{NumericAl RESUlts}

A comparison of the simulation and analytical results of 4PAM modulation for various $N$ values and Rayleigh fading channels obtained by evaluating (8), is shown in Figure 1. The closed-form expression (8) is seen to be in good agreement with the Monte Carlo simulation results. Equation (8) is general and can be used for the error probability evaluation of any Gray coded MPAM signal constellation set. As another 


$$
\begin{aligned}
P_{b}(h)= & K_{M} \sum_{k=1}^{\log _{2}(M)} \sum_{i=0}^{\mathcal{L}(k, M)} \mathcal{X}(k, i, M) \cdot Q\left(\sqrt{M \gamma}\left(\frac{2 \cdot h \cdot \mathcal{Y}(i)+(M-1) \cdot 2^{-N+1}}{\sqrt{2 S_{M}}}\right)\right) \cdot\left(1+\frac{h \cdot \mathcal{Y}(i) \cdot 2^{N}}{M-1}\right)+ \\
& K_{M} \sum_{k=1}^{\log _{2}(M)} \sum_{i=0}^{\mathcal{L}(k, M)} \mathcal{X}(k, i, M) \cdot Q\left(\sqrt{M \gamma}\left(\frac{2 \cdot h \cdot \mathcal{Y}(i)-(M-1) \cdot 2^{-N+1}}{\sqrt{2 S_{M}}}\right)\right) \cdot\left(1-\frac{h \cdot \mathcal{Y}(i) \cdot 2^{N}}{M-1}\right)- \\
& K_{M} \sum_{k=1}^{\log _{2}(M)} \sum_{i=0}^{\mathcal{L}(k, M)} \frac{\mathcal{X}(k, i, M) \cdot \sqrt{S_{M}}}{(M-1) \sqrt{M \pi \gamma} 2^{-N+1}} \cdot\left[e^{-\frac{M \gamma}{S_{M}}\left(h \cdot \mathcal{Y}(i)+(M-1) \cdot 2^{-N}\right)^{2}}-e^{-\frac{M \gamma}{S_{M}}\left(h \cdot \mathcal{Y}(i)-(M-1) \cdot 2^{-N}\right)^{2}}\right] .
\end{aligned}
$$

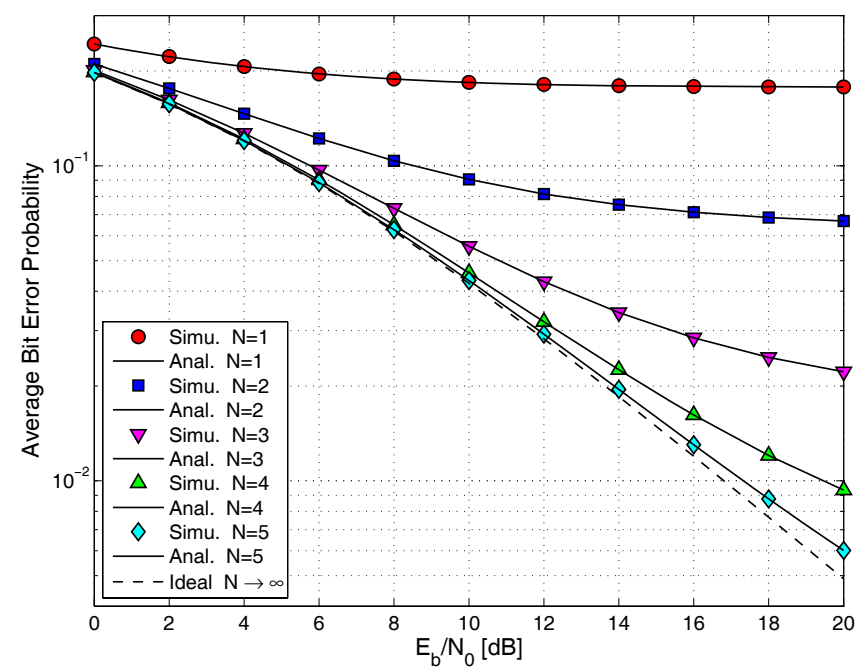

Fig. 1. A comparison of simulation and analytic results of 4PAM for various $N$ values over a Rayleigh fading channel (i.e., $m=1$ ).

interesting application of the obtained results, the impact on BER by changing the ADC resolution of 2PAM for different $m$ parameters at $E_{b} / N_{0}=10 \mathrm{~dB}$ is depicted in Figure 2. This can help the system designer to quickly establish the degradation in system performance due to limited ADC resolution for a given channel ( $m$ parameter). For example, a maximum BER of $10^{-3}$ at $E_{b} / N_{0}=10 \mathrm{~dB}$ can be achieved with a 2 bit ADC at $m=7$, while for a channel with $m=5$ a 3 bit ADC would be required.

\section{CONCLUSIONS}

In this letter, the BER of MPAM modulation schemes in the presence of ADC QN has been analyzed for fading and non-fading scenarios. Closed form expressions for the BER of Gray coded MPAM signal constellations in the presence of AWGN, Nakagami fading and ADC QN, are presented. These expressions are then used to investigate the system performance. This analysis enables the system designer to rapidly determine for example the minimum number of bits required to achieve a desired performance in a particular fading scenario.

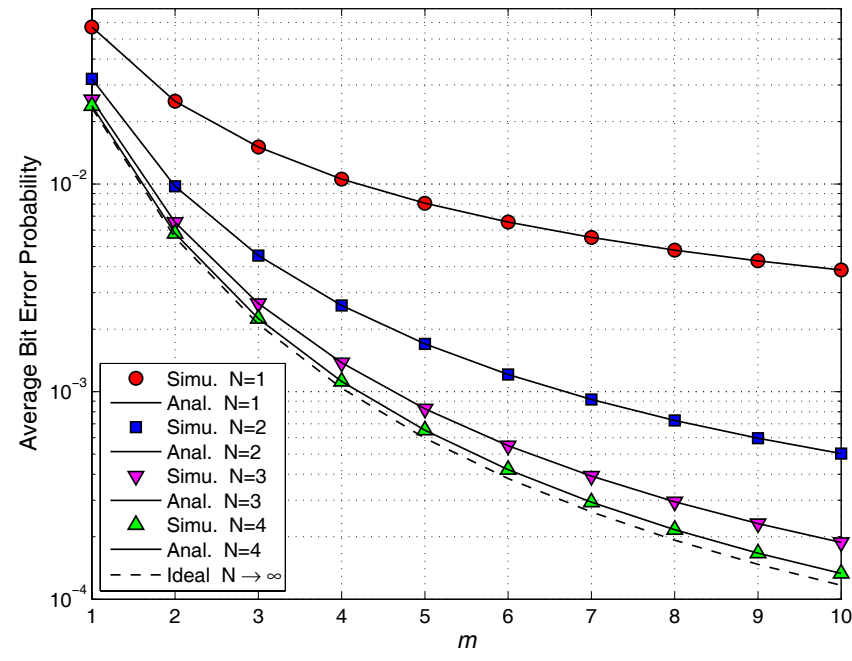

Fig. 2. BER of 2PAM as function of various $N$ and $m$ values over Nakagami$m$ fading channels at $E_{b} / N_{0}=10 \mathrm{~dB}$.

\section{ACKNOWLEDGMENTS}

This work was supported by IOP GenCom under SiGi Spot project IGC.0503.

\section{REFERENCES}

[1] J. Tubbax, B. Come, L. Van der Perre, S. Donnay, M. Engels, H. De Man, and M. Moonen, "Compensation of IQ imbalance and phase noise in OFDM systems," IEEE Trans. Wireless Commun., vol. 4, no. 3, pp. 872-877, May 2005.

[2] T. Matsumoto, S. Fujita, H. Lei, M. Sim, and Y. Z. Raymond, "Panasonic PHY and MAC proposal to IEEE802.15 TG3c CFP," 15-07-0698-05003c-panasonic-ook-based-60ghz-phy-mac-proposal.pdf, July 2007. [Online]. Available: https://mentor.ieee.org/802.15/file/07/.

[3] A. Moschitta and D. Petri, "Wideband communication system sensitivity to overloading quantization noise," IEEE Trans. Instrumentation and Measurement, vol. 52, no. 4, pp. 1302-1307, Aug. 2003.

[4] J. H. Reed, Software Radio: A Modern Approach to Radio Engineering. Prentice Hall, 2002.

[5] K. Cho and D. Yoon, "On the general BER expression of one- and twodimensional amplitude modulations," IEEE Trans. Commun., vol. 50, no. 7, pp. 1074-1080, July 2002.

[6] R. K. Bock and W. Krischer, The Data Analysis BriefBook. Berlin, Germany: Springer-Verlag, 1998.

[7] M. Abramowitz and I. A. Stegun, Handbook of Mathematical Functions with Formulas, Graphs and Mathematical Tables, 9th ed. New York: Dover Publications, 1970. 